\title{
TELA EXCLUIDORA DE RAINHA NA PRODUÇÃO DE MEL E NA LONGEVIDADE DAS OPERÁRIAS EM COLMEIAS DE Apis mellifera
}

\author{
THE QUEEN EXCLUDER IN HONEY PRODUCTION AND LONGEVITY OF WORKERS \\ IN BEEHIVES OF WORKERS IN BEEHIVES OF Apis mellifera
}

\author{
Alexandre Rusig ${ }^{1}$ Regina Helena Nogueira-Couto ${ }^{2}$ Leomam Almeida Couto ${ }^{3}$
}

\section{RESUMO}

\begin{abstract}
Este trabalho tem como objetivos verificar as áreas de cria e alimento e a longevidade de operárias em colmeias de Apis mellifera, em apicultura fixa (mata) e migratória (laranja e eucalipto), sem $\left(T_{1}\right)$ e com $\left(T_{2}\right)$ tela excluidora de rainha. Foram utilizadas colmeias modelo Langstroth, com sobrecaixa. Na apicultura fixa, estas colmeias foram analisadas durante 476 dias e mapeadas a cada 30 a 45 dias para obtenção das áreas de cria e alimento. Na migratória, as colmeias foram analisadas antes $e$ após a florada. Foi estudada também a longevidade das operárias, nos dois tratamentos. Os dados mostraram que em fluxo baixo de néctar (apicultura fixa, em mata), o uso de tela excluidora apresentou maior eficiência na separação do mel das crias. Entretanto, quando o fluxo de néctar foi alto (floradas de laranja e eucalipto), a tela excluidora não foi eficiente, pois as abelhas rapidamente preencheram os favos disponiveis no ninho inferior, misturando-o com cria. Observou-se também redução na longevidade das operárias das colmeias com tela excluidora, em dois dos três testes realizados. Este trabalho, desenvolvido em três locais diferentes, com plantas apícolas distintas evidenciou também a variabilidade e a grande influência ambiental no desenvolvimento das colônias de Apis mellifera.
\end{abstract}

Palavras-chave: tela excluidora, mel, Apis mellifera, abelhas.

\section{SUMMARY}

This work was conducted to verify the brood and food area and the longevity of the workers in beehives of Apis mellifera, in established (in woods) and migratory (orange and eucalyptus) beekeeping, without $\left(T_{1}\right)$ and with $\left(T_{2}\right)$ queen excluders. In the wood, the beehives were analyzed for 476 days and mapped at intervals of 30 to 45 days to obtain the areas with brood, honey, and pollen. In the migratory, the beehives were analyzed before and after bloom. The longevity of the workers was also studied, in both treatments. The data showed that in low flow of nectar (in woods), the queen excluder demonstrated to be efficient allowing a largest separation of the honey and brood. However, when the nectar flow was high (orange and eucalyptus), the queen excluder was not efficient, due to the quicky filling out of the available combs with honey in the inferior nest, mixing it with its brood. Reduction in the longevith of the workers was also observed in $T_{2}$, in 2 of 3 tests. This work, accomplished at three different places, with different plants, evidenced the variability and the great environmental influence in the development of the colonies of Apis mellifera.

Key words: queen excluder, honey, Apis mellifera, honeybees.

\section{INTRODUÇÃO}

A tela excluidora de rainhas de Apis mellifera L. foi desenvolvida por Abbé Collin em 1869, na França (MORSE \& HOOPER, 1985). Entretanto, segundo TABER (1986), só começou a ser fabricada e comercializada em 1905, por Market.

As telas excluidoras podem ser feitas de arame, plástico ou placas de alumínio perfuradas. $\mathrm{O}$ princípio de funcionamento se baseia na existência de frestas ou orifícios que dão passagem às abelhas operárias mas não aos zangões e à rainha. Desenvolvida com o objetivo de confinar a rainha de Apis mellifera em partes da colmeia, permite o livre trânsito das operárias, podendo ser utilizada em muitas aplicações. Por meio dela, pode-se controlar as atividades da rainha e, em conseqüência, o desenvolvimento e configuração da colmeia (PATERSON, 1952; TREDWELL, 1968; ROOT, 1977; MORSE, 1980). Utilizada na frente do alvado

\footnotetext{
${ }^{1}$ Zootecnista, Bolsista da FAPESP, Processo no 90/3411-8.

${ }^{2}$ Biólogo, Professor Titular, Departamento de Zootecnia, Faculdade de Ciências Agrárias e Veterinárias, Universidade Estadual Paulista, Rodovia Prof. Paulo Castellane s/n, 14870-000, Jaboticabal, SP.

${ }^{3}$ Zootecnista, Professor Adjunto, Departamento de Zootecnia da FAFRAM, Ituverava (SP).
} 
dificulta a atividade de enxameação ou a saída da rainha para o vôo nupcial, quando é necessário o controle de seu acasalamento. É essencial na produção de rainhas e na exploração comercial da geléia real. Entretanto, seu uso na produção comercial de mel ainda suscita controvérsias. Apesar de a tela excluidora já ter sido adotada como um equipamento apícola de grande utilidade por alguns apicultores, pouco se sabe sobre seus efeitos na produção de mel e sobre a longevidade das operárias.

Alguns produtores afirmam que o uso da tela excluidora, entre o ninho e a melgueira, aumenta a produção exclusiva de mel em favos (BRAUN, 1951; SMITH, 1965); outros consideram que o seu uso cria dificuldades no desenvolvimento da colmeia, reduzindo a produção de mel, a postura da rainha (TABER, 1986) afetando a longevidade das operárias por danificar as suas asas (CAILLAS, 1963), além de causar custos adicionais e perda de tempo nas revisões (MORSE \& HOOPER, 1985).

Considerando o exposto, este trabalho tem como objetivos estudar a influência do uso da tela excluidora de rainhas, entre o ninho e a melgueira, na produção de cria e de alimento e na longevidade de operárias de colmeias de apiários comerciais instalados em floradas de mata nativa, eucalipto e laranja.

\section{MATERIAL E MÉTODOS}

O experimento foi conduzido em colmeias instaladas em três floradas, em épocas diferentes. Inicialmente, os dados foram coletados de colmeias instaladas em um remanescente de floresta, com área de 22 hectares, localizado na região de Jaboticabal (SP), no período de 24/04/90 a 13/08/91 (476 dias). Posteriormente, os dados foram coletados em colmeias instaladas em 30 hectares de laranjeiras, na região de Lins (SP), Fazenda São Miguel, de 14/08/92 a 29/11/92 (107 dias). Finalmente, coletaram-se dados de colmeias instaladas em área de reflorestamento com eucalipto, próxima à cidade de Luiz Antônio (SP), de 17/04/93 a 07/05/93 (20 dias).

Em todos os locais e floradas, utilizaramse 10 colmeias de Apis mellifera, instaladas em ninhos modelo Langstroth. As colmeias eram distribuídas em dois tratamentos. No tratamento 1 $\left(\mathrm{T}_{1}\right)$, cinco colmeias permaneceram sem tela excluidora de rainhas. No tratamento $2\left(\mathrm{~T}_{2}\right)$, cinco colmeias receberam tela excluidora de rainhas, confeccionadas em arame liso, colocadas entre o ninho e a melgueira. Todas as colmeias foram inicialmente homogeneizadas quanto às áreas de cria e alimento, receberam rainhas irmãs, da mesma idade e fecundadas no ar, e foram distribuídas, aleatoriamente, entre os tratamentos.

No decorrer do experimento, foram realizadas avaliações periódicas das áreas de cria e de alimento presente nas colmeias. Essas avaliações eram feitas mapeando-se individualmente todos os favos das colmeias, de acordo com o método desenvolvido por AL-TIKRITY et al. (1971). Durante a permanência na área de mata nativa, realizaram-se onze mapeamentos, em todas as colmeias. Nas floradas da laranja e de eucalipto, os mapeamentos foram realizados no início e no final do período experimental (dias 13/08/92 e 29/11/92 e 17/04/93 e 07/05/93.

Foi estudada, também, a longevidade de operárias irmãs introduzidas em colmeias sem $\left(\mathrm{T}_{1}\right)$ e com a tela excluidora $\left(\mathrm{T}_{2}\right)$, de acordo com o método proposto por SAKAGAMI \& FUKUDA (1968). As operárias utilizadas nasceram de favos de crias retirados de uma mesma colmeia e colocados em estufa com temperatura e umidade relativa controladas. Assim que emergiam, as abelhas recebiam uma pequena placa circular colorida, numerada de 0 a 99, que era colada no seu tórax e introduzida numa colmeia sem tela excluidora $\left(\mathrm{T}_{1}\right)$ ou com tela excluidora $\left(\mathrm{T}_{2}\right)$. Este teste foi realizado com 100 operárias, por tratamento, em seis colmeias diferentes, num total de 600 operárias analisadas, 300 para $T_{1}$ e 300 para $T_{2}$. Diariamente, essas colmeias eram abertas, e a presença de cada uma das operárias numeradas era registrada.

Os dados foram submetidos a análises de variância, com delineamento inteiramente casualizado, no programa AVCTT, que inclui o teste de Tukey, para comparação das médias e correlação parcial.

\section{RESULTADOS E DISCUSSÃO}

\section{Mata nativa}

A tabela 1 apresenta os dados obtidos nas colmeias do tratamento $\mathrm{T} 1$ (sem o uso de tela excluidora de rainhas) e $\mathrm{T}_{2}$ (com tela excluidora), durante o período em que permaneceram numa área de mata. Os onze mapeamentos realizados no período mostraram que tanto a área de cria (ovolarva e pupa), de alimento (mel e pólen) e a área total (cria e alimento) foram superiores nas colmeias com tela excluidora, em relação às sem tela.

Apesar das flutuações observadas durante o período (476 dias), para os dois tratamentos, 
Tabela 1 - Dados médios das áreas $\left(\mathrm{cm}^{2)}\right)$ de cria e de alimento de 11 mapeamentos de 10 colônias desenvolvidas numa mata, no período de 24/04/90 a 13/08/91, sem $\left(T_{1}\right)$ e com $\left(T_{2}\right)$ tela excluidora de rainha entre o ninho e a sobrecaixa. $\mathrm{O}$ desvio padrão encontra-se entre os parênteses.

\begin{tabular}{ccccccccc}
\hline \multirow{2}{*}{ TRATAMENTOS } & \multicolumn{2}{c}{ Operárias } & \multicolumn{2}{c}{ Zangão } & \multirow{2}{*}{ Mel } & \multirow{2}{*}{ Pólen } & \multirow{2}{*}{ Total } \\
\cline { 2 - 6 } & & Ovo-larva & Pupa & Ovo-larva & Pupa & & & \\
\hline \multirow{4}{*}{ NI } & 1309,5 & 1780,6 & 25,4 & 35,8 & 3156,8 & 774,2 & 7082,3 \\
& & $(622,2)$ & $(931,4)$ & $(70,5)$ & $(69,8)$ & $(2005,2)$ & $(557,8)$ & \\
T1 & SC & 40,8 & 63,9 & 6,5 & 5,6 & 610,6 & 12,3 & 739,7 \\
& & $(193,0)$ & $(238,8)$ & $(35,4)$ & $(26,1)$ & $(1328,1)$ & $(53,5)$ & \\
& & 1350,3 & 1844,5 & 31,9 & 41,4 & 3767,4 & 786,5 & 7822,0 \\
& TI & 1440,4 & 2109,5 & 49,7 & 39,5 & 3138,1 & 1408,7 & 8185,9 \\
& & $(798,0)$ & $(1171,2)$ & $(155,6)$ & $(74,1)$ & $(1508,5)$ & $(740,7)$ & \\
T2 & SC & Zero & Zero & Zero & Zero & 1847,7 & Zero & 1847,7 \\
& & & & & & $(3090,3)$ & & \\
& T & 1440,4 & 2109,5 & 49,7 & 39,5 & 4985,8 & 1408,7 & 10033,6 \\
\hline
\end{tabular}

$\mathrm{NI}=$ Ninho inferior, $\mathrm{SC}=$ sobrecaixa, $\mathrm{T}=$ Área total.

notou-se que nos meses mais frios do ano, ocorreram quedas bruscas da área total utilizada, especialmente para o tratamento $\mathrm{T}_{1}$. Por outro lado, durante a primavera e o verão, ambos os tratamentos apresentaram a maior produção. Considerando todo o período, $\mathrm{T}_{2}$ apresentou, em média, $11,4 \%$ a mais de área total de cria de operária que $T_{1}$.

Quanto ao desenvolvimento de cria de zangões, observaram-se três picos de produção (abril/90, outubro/90 e maio/91), coincidentes nos dois tratamentos $\left(\mathrm{T}_{1}\right.$ e $\left.\mathrm{T}_{2}\right)$. Entretanto, em $\mathrm{T}_{2}$, esses picos foram menos acentuados que os obtidos em $\mathrm{T}_{1}$, apesar da média geral (em todo o período) da área de cria de zangão de $\mathrm{T}_{2}$ ser superior a $\mathrm{T}_{1}(89,2+/-81,8$ e $73,3+/-65,2 \mathrm{~cm}^{2}$, respectivamente), ao nível de $5 \%$. Esses dados mostram que a presença da tela não reduz, necessariamente, a produção de zangões. No presente estudo, notou-se o inverso, ou seja, as colônias do tratamento com tela apresentaram maior área média com crias de zangões.

Por outro lado, o uso da tela mostrou ser eficaz para impedir a presença de cria de zangões nas sobrecaixas, apesar de que, dos onze mapeamentos realizados, somente em um deles (23/02/91) constatou-se a presença de crias de zangões e pólen na sobrecaixa de duas colmeias do tratamento $T_{1}$, totalizando $844 \mathrm{~cm}^{2}$ de área utilizada. Nessa data, verificou-se ainda a maior produção média de mel de todo o período. A análise de correlação parcial mostrou que a área de cria de zangões está diretamente relacionada com a área de alimento total, obedecendo à seguinte equação:

cria de zangões $=10,32+0,005 \mathrm{Y}$, em que $\mathrm{Y}$ é equivalente à área total de alimento.
As análises estatísticas mostraram que a produção de alimento (mel e pólen) foi superior para o tratamento $\mathrm{T}_{2}$ (com tela) com relação à $\mathrm{T}_{1}$ (sem tela), ao nível de 5\%. As médias da área de pólen estocado em $\mathrm{T}_{1}$ e $\mathrm{T}_{2}$ foram, respectivamente, 786,5 +/611,3 e 1408,7 +/- 740,7; sendo $\mathrm{T}_{2}, 79,1 \%$ superior à $\mathrm{T}_{1 .}$

As médias das áreas de mel nos tratamentos $\mathrm{T}_{1}$ e $\mathrm{T}_{2}$ foram, respectivamente, 3767,4 +/- 1611,3 e $4985,8+/-\quad 2666,1$. Isso mostra que as colmeias do tratamento $T_{2}$ armazenaram, no ninho e no sobreninho, $32,3 \%$ mais mel durante o experimento que as colmeias de T1. Considerando somente as áreas de mel nas sobrecaixas dos dois tratamentos, observa-se $610,6+/-1328,1$ e $1847,7+/-3090,3 \mathrm{~cm}^{2}$ para $\mathrm{T}_{1}$ e $\mathrm{T}_{2}$, respectivamente, sendo $\mathrm{T}_{2} 202,6 \%$ superior à $\mathrm{T}_{1}$. A produção de mel no ninho inferior não apresentou diferenças significativas nos dois tratamentos, sendo suas médias 3156,8 +/- 2005,2 e 3138,1 +/$1508,5 \mathrm{~cm}^{2}$ para $T_{1}$ e $T_{2}$, respectivamente. Pelos dados obtidos, nota-se que, nas colônias com tela excluidora $\left(T_{2}\right)$, as abelhas aproveitaram a área do ninho para cria e mel, estocando nesse compartimento igual quantidade de mel que as colônias sem tela. No entanto, depositaram maior quantidade de mel nos favos da sobrecaixa.

Considerando o total de mel armazenado, nota-se que $\mathrm{T}_{1}$ (sem tela) e $\mathrm{T}_{2}$ (com tela) estocaram 83,8 e $62,9 \%$ no ninho inferior e 16,2 e $37,1 \%$ no superior, respectivamente. Isso mostra que a presença da tela excluidora afetou a distribuição do mel. Na sua ausência, ocorreu maior depósito no ninho inferior, onde o mel fica distribuído nos quadros, próximo as áreas de cria.

\section{Florada de laranjeiras}

As tabelas 2 e 3 apresentam os dados dos dois mapeamentos realizados, respectivamente, na introdução e na retirada das colmeias do laranjal. Os dados obtidos mostraram aumentos no segundo mapeamento tanto na área total de crias de operárias, como na área de cria de zangões em $\mathrm{T}_{1}$ (sem tela) e $\mathrm{T}_{2}$ (com tela), em função do aumento na disponibilidade de alimentos.

Pode-se observar que, em $\mathrm{T}_{1}$, pela ausência da tela excluidora de rainhas, $10,8 \%$ da cria de operárias e $3,9 \%$ das crias de zangões foram 
Tabela 2 - Dados médios das áreas $\left(\mathrm{cm}^{2}\right)$ de cria e de alimento de 10 colônias no início da florada da laranja, sem $\left(T_{2}\right)$ e com $\left(T_{2}\right)$ tela excluidora de rainha entre o ninho e sobrecaixa. $\mathrm{O}$ desvio padrão encontra-se entre parênteses. Data: 13/08/92.

\begin{tabular}{ccccccccc}
\hline \multirow{2}{*}{ TRATAMENTOS } & \multicolumn{2}{c}{ Operárias } & \multicolumn{2}{c}{ Zangão } & \multirow{2}{*}{ Mel } & \multirow{2}{*}{ Pólen } & Área Total \\
\cline { 2 - 6 } & & Ovo-larva & Pupa & Ovo-larva & Pupa & & & \\
\hline \multirow{2}{*}{ NI } & 1085,0 & 1360,0 & 30,5 & 14,5 & 3935,5 & 182,0 & 6607,5 \\
& & $(460,5)$ & $(892,7)$ & $(55,5)$ & $(38,4)$ & $(2146,2)$ & $(190,4)$ & \\
T1 & SC & Zero & Zero & Zero & Zero & 503,0 & Zero & 503,0 \\
& & & & & & $(1330,8)$ & & \\
& T & 1085,0 & 1360,0 & 30,5 & 14,5 & 4438,5 & 182,0 & 7110,5 \\
& NI & 884,5 & 832,5 & Zero & Zero & 4385,0 & 154,5 & 6256,5 \\
& & $(359,5)$ & $(335,3)$ & & & $(1837,0)$ & $(137,2)$ & \multirow{2}{*}{ T2 } \\
& SC & Zero & Zero & Zero & Zero & Zero & Zero & Zero \\
& T & 884,5 & 832,5 & Zero & Zero & 4385,0 & 154,5 & 6256,5 \\
\hline
\end{tabular}

$\mathrm{NI}=$ Ninho inferior, $\mathrm{SC}=$ sobre caixa, $\mathrm{T}=$ Área Total.

depositados nas sobrecaixas. Isso pode representar um transtorno realmente grande para apicultores profissionais, que trabalham com grande número de colônias. A presença de crias nos favos das melgueiras pode prejudicar ou mesmo inviabilizar a retirada do mel desses favos, trazendo prejuízos, seja na produtividade seja na qualidade do mel produzido. No tratamento $T_{2}$, a tela excluidora foi eficiente, em todas as repetições, não permitindo o acesso da rainha à sobrecaixa. Esse fato oferece subsídios para os apicultores que consideram a tela excluidora fundamental para o manejo de colmeias na produção de mel.

$\mathrm{O}$ armazenamento de pólen na melgueira ocorreu em apenas uma colônia dos dois tratamentos, mostrando que a idéia de se utilizar a tela para evitar o depósito de pólen nas imediações do mel nas sobrecaixas e o conseqüente escurecimento do mel não pode ser generalizada. Não houve diferença na quantidade de pólen estocada em $\mathrm{T}_{1}$ e $\mathrm{T}_{2}$, sendo esta área seis vezes superior no segundo mapeamento com relação ao primeiro.

Quanto ao mel, antes de ser levado às colônias para a florada da laranja, as áreas eram semelhantes nos dois tratamentos $\left(4438,5 \mathrm{~cm}^{2}\right.$ e $4385,0 \mathrm{~cm}^{2}$, para $\mathrm{T}_{1}$ e $\mathrm{T}_{2}$, respectivamente). No segundo mapeamento, essas áreas se mantiveram estatisticamente semelhantes, ao nível de 5\%. Entretanto, nota-se que a presença da tela novamente influenciou a distribuição do mel nas colmeias. $\mathrm{Na}$ sua presença, estocaram maior quantidade no ninho inferior e, em sua ausência, estocaram mais na sobrecaixa.

Do total do mel produzido, nota-se que $\mathrm{T}_{1}$ (sem tela) estocou $40,6 \%$ no ninho inferior e $59,4 \%$ na sobrecaixa, e $\mathrm{T}_{2}$ (com tela) estocou $82,7 \%$ no inferior e $17,3 \%$ na sobre caixa, o contrário do observado na mata. Esses dados mostram que, apesar de influenciar a distribuição do mel nas colmeias, não há um padrão claro de como ocorre essa influência. É possível que fluxo intenso de nectar favoreça o depósito no ninho. Uma sugestão é de se usar a tela excluidora com uma segunda entrada na sobrecaixa, o que facilitaria o depósito de mel na mesma.
Tabela 3 - Dados médios das áreas $\left(\mathrm{cm}^{2}\right)$ de cria e de alimento de 10 colônias no final da florada da laranja, sem $\left(T_{2}\right)$ e com $\left(T_{2}\right)$ tela excluidora de rainha entre o ninho e a sobrecaixa. $\mathrm{O}$ desvio padrão encontra-se entre parênteses. Data: 29/11/92.

\begin{tabular}{|c|c|c|c|c|c|c|c|c|}
\hline \multicolumn{2}{|c|}{ TRATAMENTOS } & \multicolumn{2}{|c|}{ Operárias } & \multicolumn{2}{|c|}{ Zangão } & \multirow[t]{2}{*}{ Mel } & \multirow[t]{2}{*}{ Pólen } & \multirow[t]{2}{*}{ Área total } \\
\hline & & Ovo-larva & Pupa & Ovo-larva & Pupa & & & \\
\hline \multirow{3}{*}{$\mathrm{T} 1$} & NI & $\begin{array}{l}1549,0 \\
(628,5)\end{array}$ & $\begin{array}{l}2431,0 \\
(940,8)\end{array}$ & $\begin{array}{c}125,0 \\
(139,2)\end{array}$ & $\begin{array}{r}73,5 \\
(161,3)\end{array}$ & $\begin{array}{c}2632,0 \\
(1075,9)\end{array}$ & $\begin{array}{r}1072,5 \\
(495,4)\end{array}$ & 7883,0 \\
\hline & SC & 454,2 & $\begin{array}{r}4,0 \\
(9,2)\end{array}$ & $\begin{array}{r}8,0 \\
(21,2)\end{array}$ & Zero & $\begin{array}{c}3847,5 \\
(3183,4)\end{array}$ & $\begin{array}{r}4,0 \\
(8,0)\end{array}$ & 4104,0 \\
\hline & $\mathrm{T}$ & 1789,5 & 2435,0 & 13,0 & 73,5 & 6479,5 & 1076,5 & $11.987,0$ \\
\hline \multirow{3}{*}{$\mathrm{T} 2$} & NI & $\begin{array}{l}1459,0 \\
(674,2)\end{array}$ & $\begin{array}{l}1760,0 \\
(524,9)\end{array}$ & $\begin{array}{c}86,0 \\
(127,0)\end{array}$ & $\begin{array}{c}12,5 \\
(33,1)\end{array}$ & $\begin{array}{r}4960,0 \\
(1851,2)\end{array}$ & $\begin{array}{r}895,5 \\
(482,8)\end{array}$ & 9173,0 \\
\hline & SC & Zero & Zero & Zero & Zero & $\begin{array}{c}1035,5 \\
(1547,3)\end{array}$ & $\begin{array}{l}169,0 \\
(447,1)\end{array}$ & 1204,5 \\
\hline & $\mathrm{T}$ & 1459,0 & 1760,0 & 86,0 & 12,5 & 5995,5 & 1064,5 & 10377,5 \\
\hline
\end{tabular}

$\mathrm{NI}=$ Ninho inferior, $\mathrm{SC}=$ sobrecaixa, $\mathrm{T}=$ Área total

\section{Florada de eucalipto}

Os dados obtidos no eucalipto estão registrados nas tabelas 4 e 5. Nota-se que houve aumento da área de cria de 52,9 e $58,8 \%$ para $\mathrm{T}_{1}$ e $\mathrm{T}_{2}$, respectivamente, considerando o segundo mapeamento em relação ao primeiro. Como entre os dois mapeamentos se passaram apenas 20 dias, pode-se ter uma idéia da importância dessa florada, especialmente como estimulante da postura da rainha, devido ao seu abundante fornecimento de 
Tabela 4 - Dados médios das áreas $\left(\mathrm{cm}^{2}\right)$ de cria e de alimento de 10 colônias no início da florada de eucalipto, sem $\left(\mathrm{T}_{1}\right)$ e com $\left(\mathrm{T}_{2}\right)$ tela excluidora da rainha entre o ninho e a sobrecaixa. O desvio padrão encontra-se entre parênteses. Data: 17/04/93.

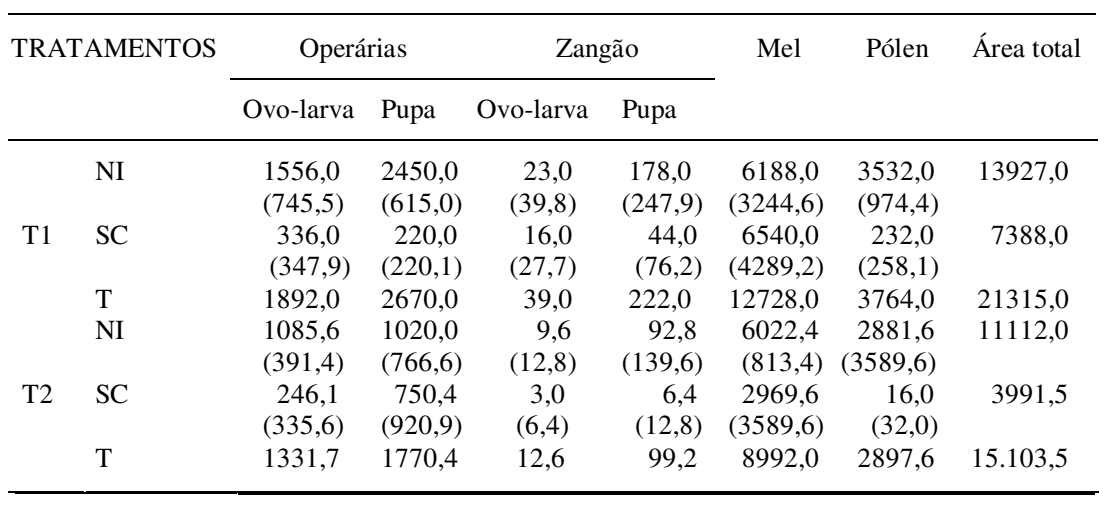

$\mathrm{NI}=$ Ninho inferior, $\mathrm{SC}=$ sobrecaixa, $\mathrm{T}=$ Área total

pólen. Observou-se um aumento de 52,1\% e 22,8\% do segundo mapeamento com relação ao primeiro na área de pólen depositada. Por sua vez, as áreas com pólen nas colônias do eucalipto foram 5,3 e 3,3 vezes superiores às obtidas nas colônias instaladas na laranja, também para $\mathrm{T}_{1}$ e $\mathrm{T}_{2}$, respectivamente. $\mathrm{Na}$ florada de eucalipto, $\mathrm{T}_{1}$ estocou $60,9 \%$ a mais de pólen que $\mathrm{T}_{2}$. Deste pólen, $\mathrm{T}_{1}$ e $\mathrm{T}_{2}$ estocaram $9,1 \%$ e $2,1 \%$ na sobrecaixa. Pode-se observar que a presença da tela excluidora não impediu a deposição de pólen nas melgueiras.

Observou-se também diferença na estocagem de mel em $\mathrm{T}_{2}$ com relação a $\mathrm{T}_{1}$, sendo que $\mathrm{T}_{1}$ estocou $23,4 \%$ a mais de mel que $\mathrm{T}_{2}$. A porcentagem de mel estocada na sobrecaixa com relação ao inferior foi equivalente a $68,0 \%$ em $\mathrm{T}_{1}$

\section{CONCLUSÕES}

(sem tela) e $42,1 \%$ em $\mathrm{T}_{2}$ (com tela), no segundo mapeamento, confirmando as observações realizadas na laranja.

Longevidade das Operárias Os dados médios obtidos referentes à longevidade das operárias, registrados na tabela 6 , mostram que, em duas repetições, o tempo de vida das operárias em $\mathrm{T}_{2}$ (com tela) foi $51,0 \%$ e $39,3 \%$ inferior à $\mathrm{T}_{1}$. Numa terceira repetição, o tempo de vida das operárias nos dois tratamentos foi semelhante. Isso mostra que o uso da tela excluidora, mesmo de arame liso, pode reduzir a longevidade das operárias.

Este trabalho, realizado em três locais diferentes com plantas apícolas distintas - flores silvestres (mata), laranja e eucalipto - mostrou que em situações de baixo fluxo de néctar, apicultura fixa em mata, a tela excluidora foi eficiente na maior separação do mel das crias, resultando em maior quantidade de mel disponível para extração pelo apicultor. Por outro lado, quando ocorreu fluxo alto de néctar, apicultura migratória na laranja e eucalipto, a tela excluidora não foi eficiente, pois as abelhas rapidamente preencheramm com mel os favos disponíveis no ninho inferior, misturando com a cria e dificultando a sua extração, oferecendo menor rendimento para o apicultor. Uma segunda entrada nas melgueiras poderia favorecer a estocagem nas sobrecaixas.

$\mathrm{O}$ estudo sobre a longevidade mostrou que, de maneira geral, o caráter é afetado pela presença da tela excluidora. Em dois dos três testes realizados, observou-se redução da longevidade das operárias nas colônias com tela excluidora (média de $45 \%$ ) com relação às sem tela.

$\mathrm{NI}=$ Ninho inferior, $\mathrm{SC}=$ sobrecaixa, $\mathrm{T}=$ Área total. 
Tabela 6 - Dados de longevidade média (dias) de operárias introduzidas em colmeias sem $\left(\mathrm{T}_{1}\right)$ e com $\left(\mathrm{T}_{2}\right)$ tela excluidora. O desvio padrão encontra-se entre os parênteses.

\begin{tabular}{lccc}
\hline Tratamentos & Maio/92 & Maio/92 & Março/92 \\
\hline \multirow{2}{*}{ T1 } & 35,2 & 32,6 & 17,4 \\
& $(21,1)$ & $(23,7)$ & $(10,7)$ \\
T2 & 23,3 & 23,4 & 17,6 \\
& $(17,7)$ & $(19,8)$ & $(11,4)$ \\
\hline
\end{tabular}

\section{REFERÊNCIAS BIBLIOGRÁFICAS}

AL-TIKRITY, W.S., HILLMANN, R.C., BENTON, A.W., et al. A new instrument for brood measurement in a honey bee colony. American Bee Journal. Hamilton, v.111, n.1, p.2021,1971 .

BRAUN, E. Shall we use queen excluder? American Bee Journal, Hamilton, v.91, n.6, p.259-260, 1951.

CAILLAS, A. La rucher de rapport. 5 ed. Giems : Chez L'Auter, 1963. 104 p.
MORSE, A.R. The complete guide to beekeeping. New York : Duttin., 1980. p.69-73.

MORSE, A.R., HOOPER, T. Encyclopedia of beekeeping., S.1.: s.e., 1985. Queen excluder: p.321.

PATERSON, C.R. Queen excluder and their use in apiary management. Journal of Agriculture, Newzeeland, v.85, n.5, p.397-398, 1952.

ROOT, A.I. ABC and XYZ of bee culture. The A. I. Medina : Root, 1977. 670p.

SAKAGAMI, S.F., FUKUDA, H. Life tables for worker honeybees. Res Popul Ecol, Tokyo, v.10, n.2, p.127-139, 1968.

SMITH, F.G. Queen excluder. Beekeeping in the tropics. 2.ed. London : Longmans, 1965. 265p.

TABER, S. The queen excluder. What it will or not do. Bee Culture, Medina, p.298-299, 1986.

TREDWELL, E.J. The Bailey queen excluder. Bee Graft, New Jersey, v.50, n.2, p.17-18, 